\title{
A New Concept of Multi-Band Carrier-less Amplitude and Phase Modulation for Bandlimited Visible Light Communications
}

\author{
Khald Werfli ${ }^{1}$, Paul Anthony Haigh ${ }^{2}$, Zabih Ghassemlooy ${ }^{1}$, Navid Bani Hassan ${ }^{1}$ and \\ Stanislav Zvanovec ${ }^{3}$ \\ ${ }^{1}$ Optical Communications Research Group, NCRLab, Faculty of Engineering and Environment, Northumbria University, Newcastle-upon-Tyne, NE1 8ST, UK \\ ${ }^{2}$ High Performance Networks Group, Faculty of Engineering, University of Bristol, BS8 1TH, UK \\ ${ }^{3}$ Department of Electromagnetic Field, Faculty of Engineering, Czech Technical University in Prague, 2 Technicka, 16627 Prague, Czech Republic
}

\begin{abstract}
In this paper, we propose a novel method of multiband carrier-less amplitude and phase ( $m$-CAP) modulation for optical wireless communication (OWC) systems. In conventional $m$-CAP systems, the total signal bandwidth is divided into $m$ equally distributed subcarriers. In this work, for the first time, we set the subcarrier bandwidths such that the first subcarrier is the same as the LED bandwidth, and subsequently distribute the remaining bandwidth equally between $m-1$ subcarriers. We show that using $m=4,6,8$ and 10 subcarriers and 16-QAM, the first subcarrier is able to achieve a bit error rate (BER) target of $10^{-4}$ with (i.e., which is below the $7 \%$ forward error correction (FEC) BER limits of $3.8 \times 10^{-3}$ ) at $E_{b} / N_{0}$ $=15.5 \mathrm{~dB}$. This is a power penalty of $\sim 1.5 \mathrm{~dB}$ to achieve the same average performance as the first five, four and three subcarriers of the conventional 10, 8 and 6-CAP and a power penalty of $\sim 1$ $\mathrm{dB}$ for new 4-CAP to achieve the average performance of the first two subcarriers of the conventional 4-CAP. Consequently, using the proposed $m$-CAP concept we show a reduction in the complexity by reducing the number of finite impulse response (FIR) filters by $80 \%, 75 \%, \sim 67 \%$ and $50 \%$ in contrast to the conventional $10,8,6$, and $4 \mathrm{CAP}$, respectively.
\end{abstract}

Keywords- Multi-band carrier-less amplitude and phase modulation; band limited VLC system

\section{INTRODUCTION}

Visible light communications (VLC) is an emerging technology that is increasingly adopted in a multitude of applications including smart cities, offices and homes, transportation, etc. [1, 2]. In contrast to traditional radio frequency (RF) based wireless communications, VLC has significant advantages including a very high unregulated $\sim 400 \mathrm{THz}$ bandwidth, high power efficiency, inherent data security, no interference and low-cost [3]. The Internet-ofThings (IoT) paradigm has led to enormous demand for high speed data transmissions. Cisco projections show that the overall mobile data traffic will increase by almost six times between 2015-2019, exceeding 24 EB/month with a compound annual growth rate of 55\% [4]. The existing RF technology is finding challenging to deal with such data traffic growth, thus resulting in a spectral congestion. The VLC technology has the potential for contributing to unlocking the spectrum congestion thus allowing the RF bandwidth been used more efficiently and effectively. The VLC technology offers multiple functionalities including illumination, data communication, indoor localization and sensing, and it will be part of future $5 \mathrm{G}$ wireless communications to offer cellular systems $[5,6]$.

However, the main bottleneck in achieving high data rate transmission using VLC is the limited modulation bandwidth $B_{\text {mod }}$ of Ce:YAG phosphor based light-emitting diodes (LEDs), which is in the region of a few $\mathrm{MHz}$ due to the slow transient response of the phosphor. Thus, in the recent years, an enormous number of studies have been carried out to overcome this limitation. One of the most popular techniques is to employ advanced modulation formats such as orthogonal frequency division multiplexing (OFDM) due to its ability to support a high number of bits/symbol, resistance to multi-path induced inter-symbol interference (ISI) and its compatibility with adaptive power- and bit-loading algorithms. Thus, applying OFDM contributes towards high transmission speed in the range of several $\mathrm{Gb} / \mathrm{s}$, but at the moment over a very short transmission span of a few centimetres [7-9]. However, employing OFDM in VLC has its own inherent issues as the high peak to average power ratio and a limited dynamic range of LEDs, which can result in clipping and subsequently signal distortion [10].

Aside from OFDM, alternative solutions have been reported in recent years to overcome the limited $B_{\text {mod. }}$. Solutions include using a dichroic optical band pass filter (BPF) at the blue wavelength in order to remove the slow phosphor response [11], and/or implementing different equalization techniques at the transmitter (Tx) and receiver $(\mathrm{Rx})[12,13]$. The problem with using equalization techniques such as the one based on the artificial neural network reported in [13] is that they are highly computationally complex. Another alternative to OFDM is the carrier-less amplitude and phase (CAP) modulation scheme, which has been reported to outperform OFDM when operating over the same physical link [14]. Transmission speeds of $1.32 \mathrm{~Gb} / \mathrm{s}$ and $1.08 \mathrm{~Gb} / \mathrm{s}$ at a BER of $10^{-3}$ were achieved with CAP and OFDM, respectively, indicating an improvement of $\sim 20 \%$. CAP is similar to quadrature amplitude modulation (QAM), where the data is transmitted in the form of two orthogonal waveforms in-phase and quadrature components. However, CAP does not 
use a local oscillator, instead uses pulse shaping filters to generate the two signals and realize the time-domain orthogonality by designing the filters as a Hilbert pair. However, employing CAP in VLC requires a high gain and flat frequency response, which rarely exists in VLC due to the aforementioned band-limitations, which cause the first order low pass filter (LPF) behaviour with $20 \mathrm{~dB} /$ decade attenuation outside the $-3 \mathrm{~dB}$ shoulder.

Recently, in [15], for the first time in VLC, the concept of multi-band CAP ( $m$-CAP) modulation format was experimentally demonstrated. The signal bandwidth was split into $m$ subcarriers (SCs) in order to relax the requirement of the flat frequency response. The report in [15] proposed that the signal attenuation per equally spaced SCs is less than that of a single SC over the entire allocated signal bandwidth. In the tested system, the conventional CAP signal was divided equally into $m=\{2,4,6,8,10\}$ SCs over a fixed total signal bandwidth $B$ of $6.5 \mathrm{MHz}$. Dividing into $m$-SCs led directly to an increase in the signal to noise ratio (SNR) per SC for increasing $m$, thus, allowing bit-loading. The SNR was estimated via the error vector magnitude (EVM) of a binary phase shift keying (BPSK) pilot for each SC, before updating each subcarrier with the appropriate number of bits/symbol. It was shown that by increasing the number of SCs, higher transmission speeds (spectral efficiency) of $31.53 \mathrm{Mb} / \mathrm{s}(4.85$ $\mathrm{b} / \mathrm{s} / \mathrm{Hz}$ ) could be achieved for $m=10$. However, increasing the number of SCs leads to the growth of the required number of pulse shaping FIR filters both at the Tx and Rx and hence, a substantial rise in the overall system computational complexity.

In this work, motivated by the relationship between the improved performance and increased complexity, we introduce a new concept of $m$-CAP where instead of dividing the subcarriers equally, we set the first subcarrier bandwidth to be equal to the LED $B_{\text {mod }}$, and divide the remaining subcarriers equally over the remaining signal bandwidth. We normalise the signal bandwidth $B=1 \mathrm{~Hz} / \mathrm{Hz}$ in order to maintain generality, and $B_{\text {mod }}$ to $0.5 \mathrm{~B}$ in order to simulate a bandlimitation. By implementing this concept, we show that using $m=10,8,6$ and 4 , the computational complexity is reduced by $80 \%, 75 \%, \sim 67 \%$ and $50 \%$ when compared with the conventional 10,8, 6 and 4-CAP with a maximum power penalty of $\sim 1.5 \mathrm{~dB}$.

The rest of this paper is organized as follows: in Section II the system model is described, results are shown in Section III and finally conclusions are given in Section IV

\section{SYSTEM MODEL}

Fig. 1 illustrates the new concept for $m=4,6,8$ and 10 . The first SC is always set to $B_{\text {mod }}(0.5 \mathrm{~Hz} / \mathrm{Hz})$ to guarantee relaxing the flat frequency response requirement for the $1^{\text {st }} \mathrm{SC}$. The rest of $B$ is divided equally into $m-1$ equal parts as given by:

$$
B_{i}=\left\{\begin{array}{lc}
\frac{B}{2}, & i=1 \\
\frac{B-\frac{B}{2}}{m-1}, & i=2: m
\end{array} .\right.
$$

For instance, for $m=4 ; \mathrm{SC} 1=B_{\text {mod }}=0.5 \mathrm{~Hz} / \mathrm{Hz}$, while SC2, $\mathrm{SC} 3$, and SC4 are set to $0.166 \mathrm{~Hz} / \mathrm{Hz}$. Fig. 2 illustrates the schematic block-diagram of the proposed system. Firstly, for each SC a $2^{17}-1$ length pseudorandom binary sequence was generated before mapping into $M$-QAM constellations, where $M=2^{k}$ is the order of QAM modulation. Here, $k$ is a positive integer number representing the number of bits/symbol. The mapped data is up-sampled according to the number of samples of $n_{\text {samp }}$, given in [16]. The up-sampled data is then split into real $I$ and imaginary $Q$ components.

The pre-transmission signals of the transmit $I$ and $Q$ square root raised cosine (SRRC) filters are given as:

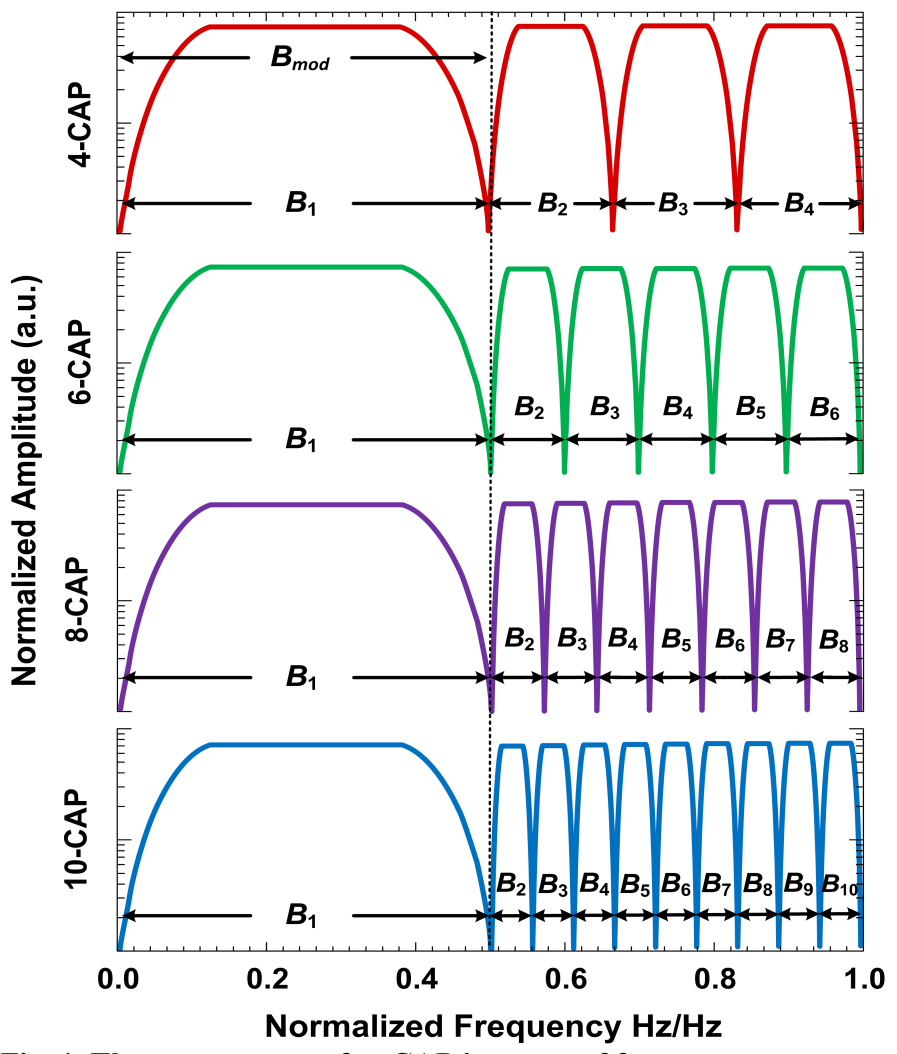

Fig. 1. The new concept of $m$-CAP in terms of frequency response for $m=4,6,8$ and 10 . It should be noted that $B_{\text {mod }}$ indicates the modulation bandwidth which set to $0.5 \mathrm{~Hz} / \mathrm{Hz}$.

$$
\begin{gathered}
S_{I, f}(t)=d_{I}(t) \otimes f_{I, T x, i}(t), \\
S_{Q, f}(t)=d_{Q}(t) \otimes f_{Q, T x, i}(t),
\end{gathered}
$$

where $d_{I}(t)$ and $d_{Q}(t)$ are the $I$ and the $Q$ baseband data, respectively and $\otimes$ denotes convolution. Also, $f_{I, T x, i}(t)$ and $f_{Q, T x, i}(t)$ denote the impulse response (IR) of the $I$ and $Q$ SRRC filters of each $i^{\text {th }} \mathrm{SC}$, respectively, defined as:

$$
\left[\frac{f_{I, T x, i}(t)=\cos \left(2 \pi f_{c, i} t\right) .}{\frac{\sin \left(\frac{\pi t}{T_{S}}(1-\beta)\right)+4 \beta \frac{t}{T_{S}} \cos \left(\frac{\pi t}{T_{S}}(1+\beta)\right)}{T_{S}}\left(1-\left(4 \beta \frac{t}{T_{S}}\right)^{2}\right)}\right]
$$



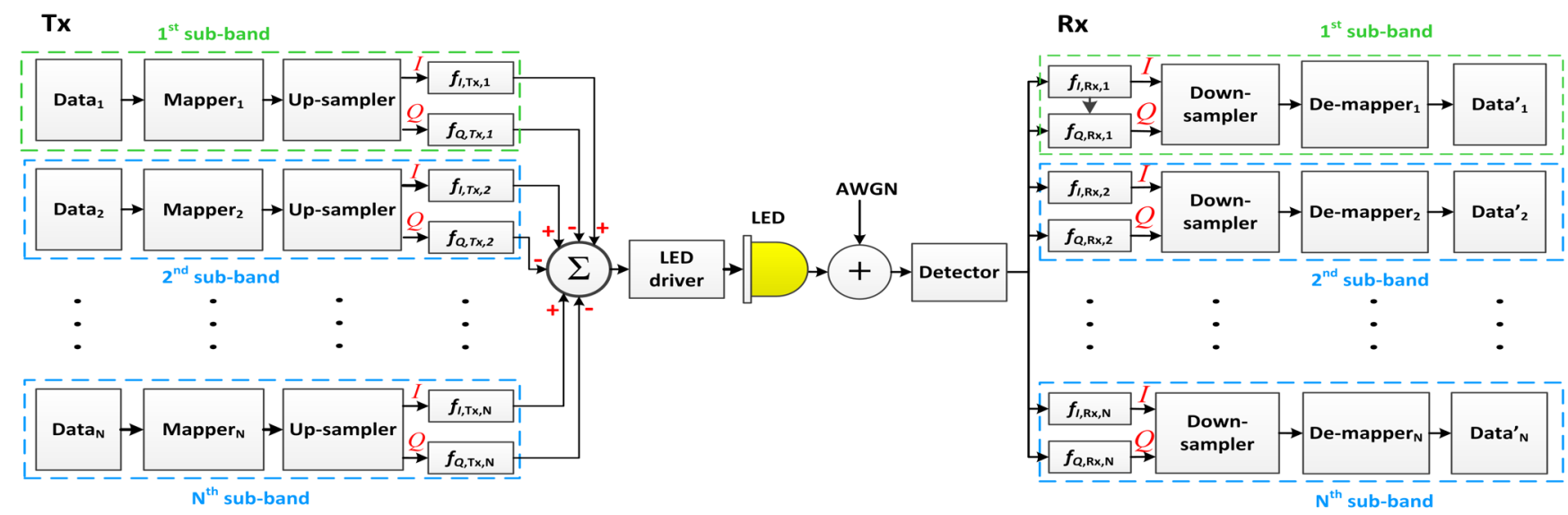

Fig. 2. The schematic block diagram of the proposed $m$-CAP system.

$$
\begin{gathered}
f_{Q, T x, i}(t)=\sin \left(2 \pi f_{c, i} t\right) . \\
{\left[\frac{\sin \left(\frac{\pi t}{T_{s}}(1-\beta)\right)+4 \beta \frac{t}{T_{s}} \cos \left(\frac{\pi t}{T_{s}}(1+\beta)\right)}{\frac{\pi t}{T_{s}}\left(1-\left(4 \beta \frac{t}{T_{s}}\right)^{2}\right)}\right]}
\end{gathered}
$$

where $T_{s}, \beta$, and $i$ denote the sampling time, SRRC filter rolloff factor and the number of SCs, respectively. In this work $\beta$ is set to 0.15 for consistency with the literature [16]. Here, $f_{c, i}$ is the frequency of the $i^{\text {th }} \mathrm{SC}$ given as:

$$
f_{c, i}=\left\{\begin{array}{cl}
\frac{B_{\text {mod }}}{2} & i=1 \\
\frac{B-B_{\text {mod }}}{2(m-1)}(2 i-3)+\frac{B_{\text {mod }}}{2} & i=2: m
\end{array} .\right.
$$

The final waveform of transmission is found by subtracting the pre-transmission signals as follows:

$$
S(t)=S_{I, f}(t)-S_{Q, f}(t) .
$$

The signal is then used to intensity modulate the LED, which is modelled as an ideal analogue LPF with a $B_{\text {mod }}=0.5$ $\mathrm{Hz} / \mathrm{Hz}$ as mentioned. In the channel, an additive white Gaussian noise (AWGN) is added to the transmitting signal and the signal is then detected by a photodiode at the receiver side. The detected signal is passed to the time-reversed inphase $f_{I, R x, i}(-t)$ and quadrature $f_{Q, R x, i}(-t)$ SRRC filters following down-sampling. Finally, the constellation is demapped. In order to determine the BER, a symbol-by-symbol calculation is performed.

In this paper, the BER target is set at $10^{-4}$ to allow enough headroom for the $7 \%$ FEC BER limit of $3.8 \times 10^{-3}$.

\section{RESULTS}

Here, the results of the new $m$-CAP system are presented. Also presented are the BER performance of the new and conventional $m$-CAP for $m=4,6,8$ and 10 as a function of $E_{b} / N_{0}$ when the modulation format is 16-QAM without any loss of generality. It should be noted that for the conventional $m$-CAP the SCs are divided equally across the signal bandwidth $B$ and for the proposed $m$-CAP the $1^{\text {st }} \mathrm{SC}$ has been set to $B_{\text {mod }}$ as discussed in the previous section, while the remaining subcarriers are evenly spaced between out-of-band signal bandwidth.

The performance comparison of the new $m$-CAP with the conventional $m$-CAP is discussed as follows.

\section{A. 10-CAP}

Fig. 3 (a) illustrates the performance of the new 10-CAP with the average (dot-red) performance of the first five SCs of the conventional $m$-CAP. The $1^{\text {st }} \mathrm{SC}$ has achieved the BER target of $10^{-4}$ at $E_{b} / N_{0}$ of $\sim 15.5 \mathrm{~dB}$. This BER performance is the same as the average BER performance of the first five SCs of the conventional $m$-CAP, which occupy $B_{m o d}$ with a power penalty of $\sim 1.5 \mathrm{~dB}$. Note that the first five $\mathrm{SCs}$ of the conventional $m$-CAP achieved the average BER target at $E_{b} / N_{0}$ $\sim 14 \mathrm{~dB}$. This is remarkable as it indicates that by using one SC in the proposed 10-CAP we could achieve a similar performance of the average of the first five SCs. It should be noted that for each increment of $m$-order two more pulse shaping FIR filters are needed (one for real data and one for imaginary data) at the $\mathrm{Tx}$ and following the same pattern another two FIR filters at the $\mathrm{Rx}$ are required as well. Furthermore, for $m=10,20$ FIR filters are needed each at the Tx and Rx. Thus, sacrificing a power penalty of $\sim 1.5 \mathrm{~dB}$ using the new 10-CAP the system computational complexity can be reduced by up to $80 \%$ using less number of FIR filters, which is considered as the major factor of criticism in the traditional $m$-CAP system. It should be noted that $S$ indicates the individual SCs within a given $m$, where $m$ refers to the total number of SCs in the given link. The out of $B_{\text {mod }}$ SCs in the new 10-CAP achieved the designated BER target starting form $E_{b} / N_{0} \sim 16 \mathrm{~dB}$ for the $2^{\text {nd }} \mathrm{SC}$ and it is clear that by increasing the number of SCs they experienced high frequency attenuation being outside $B_{\text {mod }}$. However, in contrast with the conventional 10-CAP they achieved slightly improved performance due to the fact that the bandwidth per SC $B_{s c}$ of outside $B_{\text {mod }}$ in the proposed 10-CAP is $B_{s c}=0.5 / 9=$ $0.055 \mathrm{~Hz} / \mathrm{Hz}$, which is less than $B_{s c}=0.1 \mathrm{~Hz} / \mathrm{Hz}$ in the 
conventional 10-CAP. As a result they offer closer approximation to the flat band response. The $10^{\text {th }} \mathrm{SC}$ converged to the BER target at $E_{b} / N_{0} \sim 20 \mathrm{~dB}$ with $\sim 8 \mathrm{~dB}$ and $\sim 4.5 \mathrm{~dB}$ power penalties from the predicted curve and the $1^{\text {st }}$ $\mathrm{SC}$, respectively. This is reflected on the constellation diagram inset in Fig. 3 (a) for a SNR of $20 \mathrm{~dB}$ of the $10^{\text {th }} \mathrm{SC}$.

B. 8-CAP

For the new 8-CAP system (Fig. 3 (b)), the $1^{\text {st }} \mathrm{SC}$ performance was the same as the new 10-CAP where it achieved the designated BER at $E_{b} / N_{0}$ of $\sim 15.5 \mathrm{~dB}$ as expected as it is always set to the $B_{\text {mod }}$. In contrast to the average performance of the first four SCs of the conventional 8-CAP $\left(B_{s c}=0.125 \mathrm{~Hz} / \mathrm{Hz}\right)$, the $1^{\text {st }} \mathrm{SC}$ showed a similar performance as of the new 10-CAP. The performance of the average of the first four SCs outperformed the $1^{\text {st }} \mathrm{SC}$ performance by $\sim 1.5$ $\mathrm{dB}$ where it achieved the BER target at $E_{b} / N_{0}$ of $\sim 14 \mathrm{~dB}$. This is reasonable as it indicates that the average of the SCs within $B_{\text {mod }}$ achieve the same SNR requirement. Implementing the new 8 -CAP system the $1^{\text {st }} \mathrm{SC}$ showed a reduction of $\sim 75 \%$ in the computational complexity compared to the $1^{\text {st }}$ four SCs in the conventional 8 -CAP when sacrificing $\sim 1.5 \mathrm{~dB}$ of power. The out of $B_{\text {mod }}$ SCs as expected experienced a high frequency attenuation imposed by the behaviour of LED, which acts as a $1^{\text {st }}$ order LPF as the inset constellation diagram in Fig. 3(b) show for the $8^{\text {th }} \mathrm{SC}$ of the new 8-CAP link.

C. 6-CAP

Fig. 3 (c) illustrates the performance of new 6-CAP, the $1^{\text {st }} \mathrm{SC}$ show a power penalty of $\sim 1.5 \mathrm{~dB}$ compared with the $1^{\text {st }}$ three $\mathrm{SCs}\left(B_{s c}=0.166 \mathrm{~Hz} / \mathrm{Hz}\right)$. Thus, showing the same performance as the new 10 and $8 \mathrm{CAP}$, but with reduced computational complexity of the system by $\sim 67 \%$. Once again the out of $B_{\text {mod }}$ SCs experienced different high frequency attenuation as the inset constellation diagram in Fig. 3 (c) at $\mathrm{SNR}=20 \mathrm{~dB}$.

\section{4-CAP}

The $1^{\text {st }} \mathrm{SC}$ of the new 4-CAP shows a lower power penalty of $\sim 1 \mathrm{~dB}$ compared with the average performance of the first two SCs $\left(B_{s c}=0.25 \mathrm{~Hz} / \mathrm{Hz}\right)$. This is because of larger $B_{s c}$ compared with $B_{s c}$ of the conventional 6, 8 and 10-CAP. However, the computational complexity of such a system is increased by only $\sim 50 \%$. The inset constellation diagram in Fig. 3 (d) confirms the attenuation experienced by the $4^{\text {th }} \mathrm{SC}$ due to the out of $B_{m o d}$ attenuation.
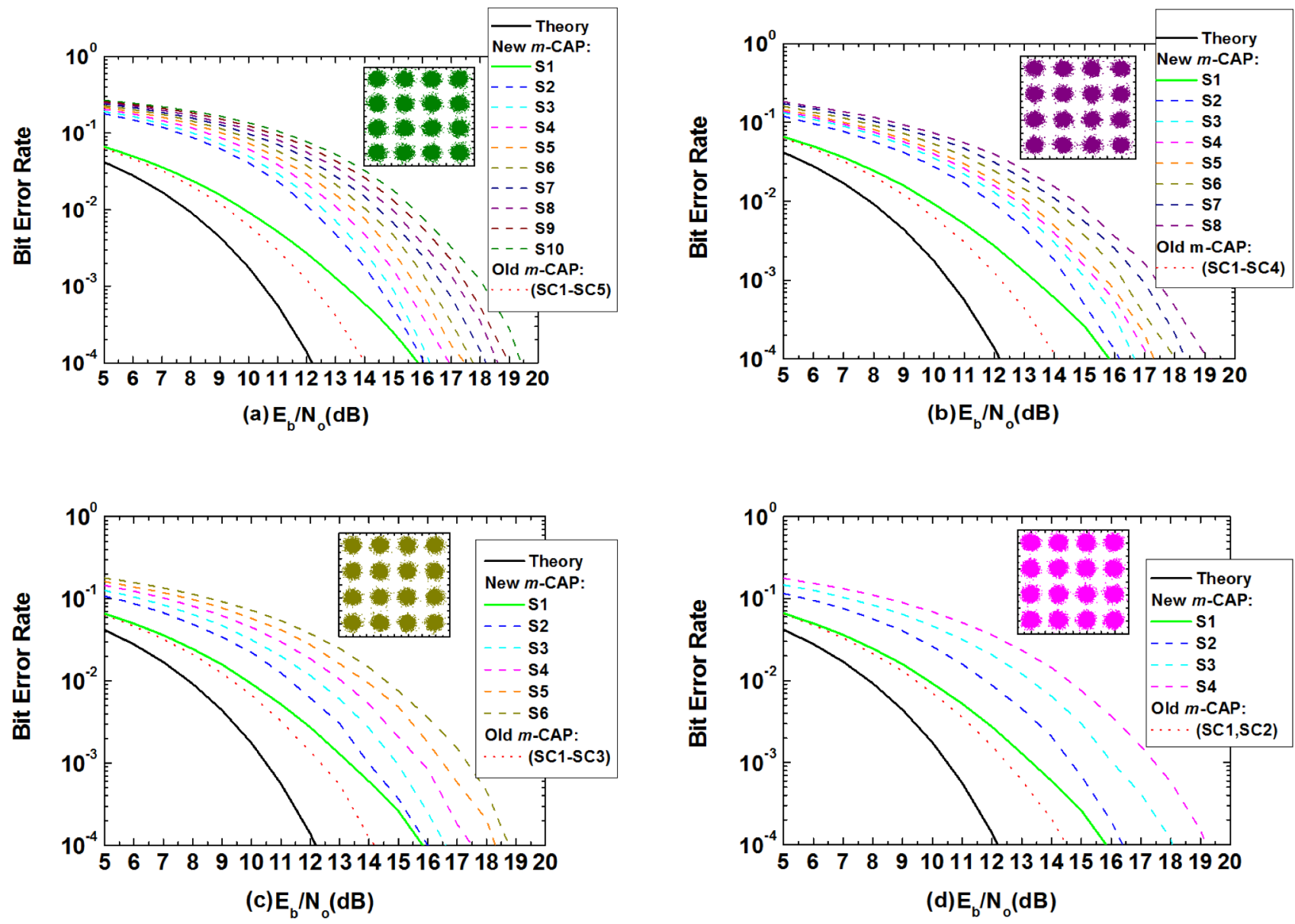

Fig. 3 (a) 16-QAM theory (solid-black), average BER performance (dot-red) of the first five SCs of the conventional 10-CAP, BER performance of the $1^{\text {st }} \mathrm{SC}$ ( solid-green) and the new 10-CAP out of $B_{\text {mod }}$ subcarriers S2-S10 (dash) as a function of $E_{b} / N_{0}$. It should be noted that $S$ indicates the individual number of SCs. (b) 16-QAM theory (solid-black), first four SCs average (dot-red) of the conventional 8-CAP, ${ }^{\text {st }}$ SC (solid-green) and 10-CAP BER performance of the out of $B_{\text {mod }}$ S2-S8 (dash) as a function of $E_{b} / N_{0}$; there is an attenuation due to the $1^{\text {st }}$ order LPF LED behaviour as clearly the inspect constellation diagram show. (c) 16-QAM theory (solid-black), average of firs three SCs (dot-red) of the traditional 6-CAP, $1^{\text {st }}$ SC (solid-green) and out of $B_{\text {mod }}$ new 6-CAP BER performance as a function of $E_{b} / N_{o}$. (d) 16-QAM theory (solid-black), first two SCs (dot-red) average BER performance of the conventional 4-CAP, $1{ }^{\text {st }}$ SC (solid-green) and the out of $B_{\text {mod }}$ S2-S4 (dash) BER performance as a function of $E_{b} / N_{0}$. The high frequency attenuation can be clearly seen from the inspect constellation diagram when $\mathrm{SNR}=20 \mathrm{~dB}$ for the last $\mathrm{SC} S 4$. 


\section{CONCLUSIONS}

In this paper, we have shown that using the new concept of $m$-CAP (i.e., $1^{\text {st }} \mathrm{SC}=B_{\text {mod, }}$, the rest of $B$ is divided equally into $m-1$ parts) at the designated BER of $10^{-4}$ the performance of the $1^{\text {st }} \mathrm{SC}$ of a high order $m=10,8$, and 6 achieved a $E_{b} / N_{0}=15.5 \mathrm{~dB}$ at a power penalty of $\sim 1.5 \mathrm{~dB}$ compared with the average performance of the first five, four and three SCs in the conventional 10, 8, and 6-CAP. For the new 4-CAP the performance of the $1^{\text {st }} \mathrm{SC}$ showed a power penalty of $\sim 1 \mathrm{~dB}$ when compared with the average performance of the first two SCs of the conventional 4CAP. Moreover, we showed a reduction in the computational complexity in the proposed 10, 8, 6 and 4 CAP by $80 \%, 75 \%, \sim 67 \%$ and $50 \%$ compared to the conventional 10, 8, 6 and 4 CAP.

\section{ACKNOWLEDGEMENTS}

One of the authors Khald Werfli has received funding from the Libyan Government toward his $\mathrm{PhD}$ study.

\section{REFERENCES}

[1] L. Grobe, A. Paraskevopoulos, J. Hilt, D. Schulz, F. Lassak, F. Hartlieb, et al., "High-speed visible light communication systems," Communications Magazine, IEEE, vol. 51, pp. 60-66, 2013.

[2] H. Burchardt, N. Serafimovski, D. Tsonev, S. Videv, and H. Haas, "VLC: Beyond point-to-point communication," Communications Magazine, IEEE, vol. 52, pp. 98-105, 2014.

[3] Z. Ghassemlooy, W. Popoola, and S. Rajbhandari, Optical wireless communications: system and channel modelling with Matlab ${ }^{\circledR}$ : CRC Press, 2012.

[4] S. Zvanovec, P. Chvojka, P. A. Haigh, and Z. Ghassemlooy, "Visible Light Communications towards 5G," Radioengineering, vol. 24, pp. 1-9, 2015.

[5] M. Yasir, H. Siu-Wai, and B. N. Vellambi, "Indoor Positioning System Using Visible Light and Accelerometer," Lightwave Technology, Journal of, vol. 32, pp. 3306-3316, 2014.

[6] C. Yew-Kiat, N. Xiao-Wei, and C. Wan-Young, "Hazardless Biomedical Sensing Data Transmission Using VLC," Sensors Journal, IEEE, vol. 13, pp. 33473348, 2013.
[8] G. Cossu, A. Wajahat, R. Corsini, and E. Ciaramella, "5.6 Gbit/s Downlink and $1.5 \mathrm{Gbit} / \mathrm{s}$ Uplink Optical Wireless Transmission at Indoor Distances ( $\geq 1.5 \mathrm{~m}$ )," in Optical Communication (ECOC), 2014 European Conference on, 2014, pp. 1-3.

[9] D. Bykhovsky and S. Arnon, "An Experimental Comparison of Different Bit-and-Power-Allocation Algorithms for DCO-OFDM," Journal of Lightwave Technology, vol. 32, pp. 1559-1564, Apr 152014.

[10] Y. Zhenhua, R. J. Baxley, and G. T. Zhou, "Peak-toaverage power ratio and illumination-to-communication efficiency considerations in visible light OFDM systems," in Acoustics, Speech and Signal Processing (ICASSP), 2013 IEEE International Conference on, 2013, pp. 5397-5401.

[11] M. Hoa Le, D. O'Brien, G. Faulkner, Z. Lubin, L. Kyungwoo, J. Daekwang, et al., "100-Mb/s NRZ Visible Light Communications Using a Postequalized White LED," Photonics Technology Letters, IEEE, vol. 21, pp. 1063-1065, 2009.

[12] W. Shao-Wei, C. Feiliang, L. Liye, H. Songlin, W. Yiguang, C. Xiaoshuang, et al., "A high-performance blue filter for a white-led-based visible light communication system," Wireless Communications, IEEE, vol. 22, pp. 61-67, 2015.

[13] P. A. Haigh, Z. Ghassemlooy, S. Rajbhandari, I. Papakonstantinou, and W. Popoola, "Visible Light Communications: $170 \mathrm{Mb} / \mathrm{s}$ Using an Artificial Neural Network Equalizer in a Low Bandwidth White Light Configuration," Journal of Lightwave Technology, vol. 32, pp. 1807-1813, 2014.

[14] F. M. Wu, C. T. Lin, C. C. Wei, C. W. Chen, Z. Y. Chen, H. T. Huang, et al., "Performance Comparison of OFDM Signal and CAP Signal Over High Capacity RGB-LEDBased WDM Visible Light Communication," Photonics Journal, IEEE, vol. 5, pp. 7901507-7901507, 2013.

[15] P. A. Haigh, A. Burton, K. Werfli, H. L. Minh, E. Bentley, P. Chvojka, et al., "A Multi-CAP Visible Light Communications System with $4.85 \mathrm{~b} / \mathrm{s} / \mathrm{Hz}$ Spectral Efficiency," IEEE Journal on Selected Areas in Communications, vol. 33, pp. 1771-1779, 2015.

[16] M. I. Olmedo, Z. Tianjian, J. B. Jensen, Z. Qiwen, X. Xiaogeng, S. Popov, et al., "Multiband Carrierless Amplitude Phase Modulation for High Capacity Optical Data Links," Journal of Lightwave Technology, vol. 32, pp. 798-804, 2014. 